\title{
Leiomiossarcoma Perineal: Relato de Caso e Revisão da Literatura
}

\section{Perineal Leiomyosarcoma: Case Report and Literature Review}

\author{
MEYLINEANDRADE LIMA'; BÁRBARA HELOISAZANCHETTAPOZZOBON ${ }^{1}$; MARCUS FABIO MAGALHÃES

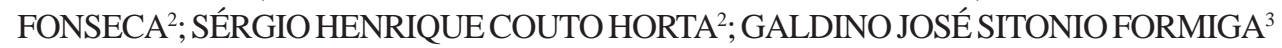 \\ 1. Residente de Coloproctologia do Hospital Heliópolis; ${ }^{2}$. Médico Assistente do Serviço de Coloproctologia do Hospital \\ Heliópolis; ${ }^{3 .}$ Chefe do Serviço de Coloproctologia do Hospital Heliópolis.
}

\begin{abstract}
LIMA MA; POZZOBON BHZ; FONSECA MFM; HORTA SHC; FORMIGA GJS. Leiomiossarcoma Perineal: Relato de Caso e Revisão da Literatura. Rev bras Coloproct, 2010;30(3): 352-355.

RESUMO: Lemiossarcomas são neoplasias malignas que se originam no músculo liso. Quando presentes na região perineal são agressivos e o tratamento cirúrgico mais adequado ainda não está bem definido. Os autores relatam o caso de uma paciente jovem, sexo feminino, com sintomatologia de nodulação perineal há oito meses. Ao exame físico apresentava abaulamento em região perineal esquerda, onde palpava-se massa fibroelástica de aproximadamente $10 \mathrm{~cm}$ de diâmetro. Ressonância nuclear magnética mostrava volumosa formação sólida de contornos regulares em região perineal à esquerda sem sinais de infiltração perilesional. $O$ tratamento realizado foi a excisão com margens amplas. A paciente encontra-se em acompanhamento ambulatorial, sem sinais de recidiva local.
\end{abstract}

Descritores: Leiomiossarcoma, Tumores retais, Períneo, Cirurgia, Radioterapia.

\section{INTRODUÇÃO}

Leiomiosarcomas (LMS) são neoplasias malignas que se originam do músculo liso ${ }^{(1)}$. São tumores raros, responsáveis por $0,7 \%$ de todas as neoplasias dos adultos ${ }^{(2)}$, constituindo $0,7-1 \%$ de todos os tumores malignos do reto e $1 \%$ dos da vulva ${ }^{(3)}$.

Eles constituem um grupo extremamente diverso de tumores, com variações histológicas, comportamentais e de localização ${ }^{(4)}$. Quando presentes na região perineal são agressivos e o tratamento cirúrgico mais adequado ainda não está bem definido.

Os autores relatam o caso de uma paciente jovem, sexo feminino, que apresentou leiomiosarcoma perineal e foi tratada com excisão local ampla. Discutem opções terapêuticas baseadas na revisão da literatura.

\section{RELATO DO CASO}

APF, 28 anos, sexo feminino, admitida no Serviço de Coloproctologia do Hospital Heliópolis com queixa de nodulação perineal há oito meses, de crescimento lento e indolor. Negava perda ponderal, hematoquezia, mucorréia e alterações do hábito intestinal. Queixava-se de dispareunia. Era previamente hígida.

Apresentava, ao exame físico, abaulamento em região perineal à esquerda, onde se palpava massa fibroelástica, móvel, de contornos bem delimitados, medindo aproximadamente $10 \mathrm{~cm}$ de diâmetro (Figura 1). Ao toque retal sente-se massa perineal à esquerda por transmissão. Anuscopia e retosigmoidoscopia sem lesões de mucosa. Exame ginecológico com mucosa vaginal íntegra, abaulamento lateral extrínseco no terço distal. 
Ressonância nuclear magnética de pelve mostrava volumosa formação sólida (Figura 2) de contornos regulares em região perineal esquerda de aproximadamente $9 \times 7,5 \times 4,5 \mathrm{~cm}$, apresentando hipossinal em T1 e sinal intermediário em T2 com realce importante pelo meio de contraste paramagnético. A lesão rechaçava o reto e a vagina. Não se observava sinais de infiltração peri-lesional. Ao estadiamento, não apresentava sinais de lesões à distância, exame radiográfico

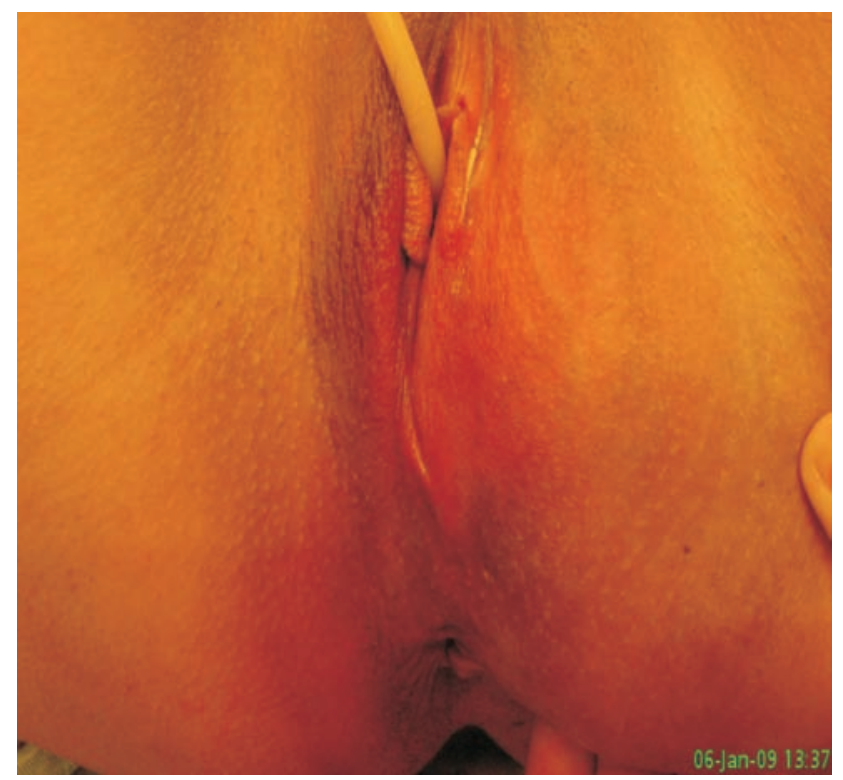

Figura 1 - Região perianal esquerda com abaulamento.

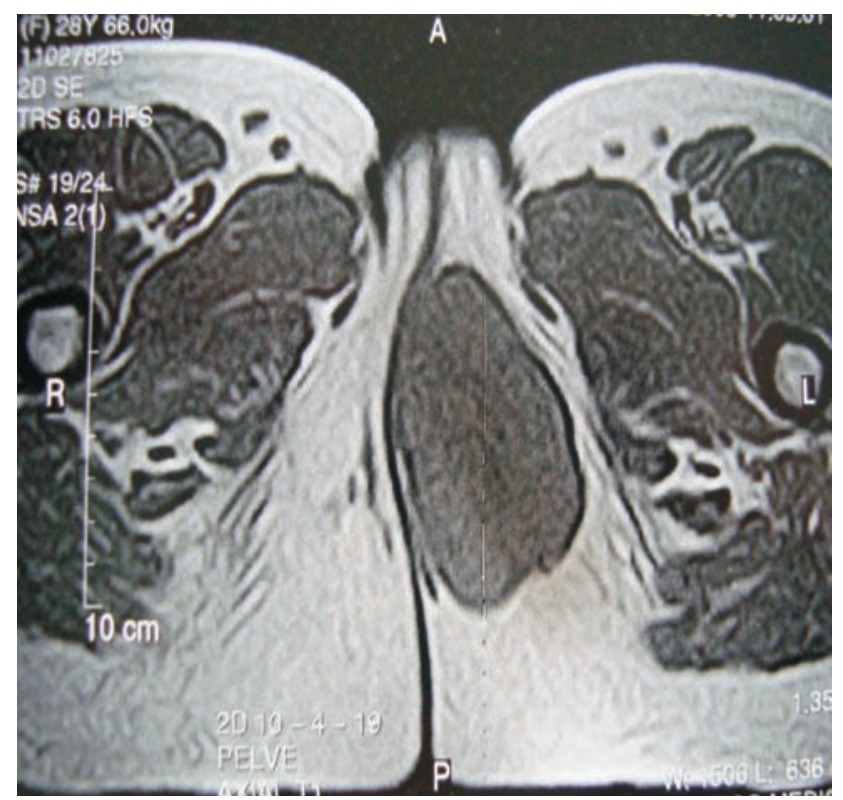

Figura 2 - RNM mostrando volumosa formação sólida à esquerda. do tórax normal e fígado sem alterações pela ultrassonografia de abdome.

Em janeiro de 2009, a paciente foi submetida à excisão local da lesão com margens amplas por via perineal sob raquianestesia. Esse procedimento foi realizado utilizando incisão longitudinal paralela à linha média sobre a lesão, com fechamento, após a exérese, por planos; não havendo necessidade de drenagem da região. O resultado do exame anátomo-patológico da peça cirúrgica revelou sarcoma de baixo grau compatível com leiomiossarcoma grau I.

A peça apresentava bordas regulares, discreta anaplasia, ausência de necrose e a presença de uma mitose por 10 campos de grande aumento. A pesquisa imuno-histoquímica (Figura 3) demonstrou a presença dos anticorpos: S100 - negativo, AML 1A 4 - positivo, AML HH F35 - positivo focal, CD- - negativo, desmina - inconclusivo, miogenina - positivo, CD 117 - positivo e AE1/AE3 - negativo; quadro imuno-histoquímico compatível com o diagnóstico de LMS.

A paciente apresentou boa evolução, recebendo alta no segundo dia de pós-operatório e encontra-se em acompanhamento ambulatorial há três meses, sem sinais de recidivas locais.

\section{DISCUSSÃO}

Os leiomiossarcomas são tumores raros que podem ocorrer em qualquer tecido muscular liso, sen-

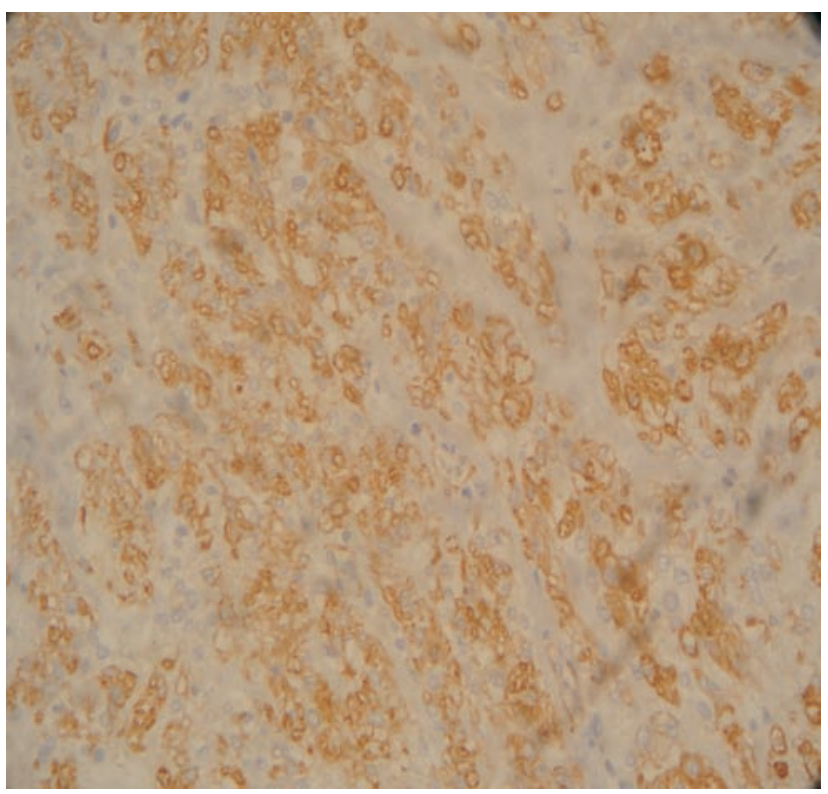

Figura 3 - Imuno-histoquímica: aumento 400x utilizando marcador actina de músculo liso. 
do mais freqüentes no estômago, intestino delgado, útero e retroperitôneo ${ }^{(4)}$. No entanto, também podem ocorrer no reto e região perineal. Acomete igualmente homens e mulheres, sendo mais comum em brancos ${ }^{(5)}$.

São classificados como superficiais (pele e tecido celular subcutâneo) e profundos (abaixo das fáscias musculares), sendo os superficiais mais comuns ${ }^{(6)}$. A apresentação típica dos LMS superficiais é de tumoração solitária, arredondada, que eleva a pele ${ }^{(1)}$, dando a impressão de serem tumores benignos ou císticos, semelhante a nossa paciente.

Se o diagnóstico de LMS é suspeitado pela história e exame físico, a tomografia computadorizada ou ressonância nuclear magnética da pelve devem ser realizadas para avaliar a extensão e invasão do tumor ${ }^{(2)}$. O diagnóstico histológico deve ser feito por biópsia, seja ela excisional para lesões pequenas (menos de $2-3 \mathrm{~cm}$ de diâmetro) ou incisional para grandes lesões. No caso em questão, não foi realizada biópsia incisional por haver suspeita de lesão benigna.

O critério mais importante para o diagnóstico dos LMS é a presença de mitoses. Haque e Dean ${ }^{(7)}$ notaram em sua série que a taxa de cinco ou mais mitoses por 50 campos de grande aumento confere potencial maligno para os tumores estromais, sendo este um dos critérios utilizados para o diagnóstico da nossa paciente. Além deste detalhe, os LMS também apresentam normalmente aderência à musculatura própria, atipia celular freqüente, e no geral são maiores que a variante benigna.

Existem poucas informações sobre a história natural e tratamento dos LMS. Segundo Rice e $\mathrm{col}^{(3)}$, o tratamento desses tumores deve ser similar ao dos sarcomas retroperitoneais, ou seja, tratamento cirúrgico agressivo. Isso pode incluir exanteração pélvica, hemipelvectomia ou amputação abdomino-perineal do reto. Dissecção linfonodal não é necessária, a menos que haja linfonodomegalia clinicamente suspeita ou biópsia linfonodal positiva.

Fields e Helwig ${ }^{(8)}$ trataram 59 pacientes com LMS cutâneo usando ressecção conservadora ou ressecção local e encontraram taxa de recorrência de $42 \%$. Entretanto, lesões recorrentes ou residuais que foram totalmente excisadas, geralmente não apresentaram recidiva local nem metástases, ou estavam relacionadas à causa da morte desses pacientes. Já para LMS subcutâneos, eles descreveram série de 12 paci- entes, onde a taxa de recidiva local foi de $50 \%$ e a taxa de metástase ou tumores relacionados à morte do paciente eram de $33 \%$, o que demonstrou uma maior agressividade para este tipo de tumor.

Khalifa e $\mathrm{col}^{(9)}$ encontraram $67,5 \%$ de recidiva local após excisão local de LMS do reto e 19,5\% após ressecção abdomino-perineal do reto. No entanto a sobrevida em cinco e dez anos foi similar. No caso da nossa paciente foi optado pela ressecção local ampla pelo fato da lesão não acometer o reto.

Radioterapia pré-operatória pode reduzir a extensão da lesão e permitir a excisão de algumas lesões previamente irressecáveis ${ }^{(10)}$. No entanto a maioria dos estudos mostram que não há aumento do controle local ou da sobrevida em pacientes que são submetidos à radioterapia adjuvante, se o tumor primário for completamene ressecado $^{(11)}$. Todavia, em lesões não completamente excisadas, Karakousis e col acreditam que a radioterapia aumente o índice de controle local ${ }^{(12)}$.

Devido à natureza infiltrativa das lesões, muitas vezes uma ressecção com margens livres é difícil durante a cirurgia, nesses casos o tratamento quimioterápico tem sido proposto. Stones e $\mathrm{col}^{(13)}$ relataram o caso de uma paciente com resposta completa após recidiva tumoral, com um esquema que associa-


usando regime com adriamicina, DTIC, vincristina e ciclofosfamida encontraram taxa de resposta de $33 \%$. Esses resultados têm encorajado o uso da quimioterapia para os pacientes com lesões recidivantes e metastáticas.

Quanto ao prognóstico, observamos que os LMS superficiais têm um melhor prognóstico que os profundos ${ }^{(1)}$. Dahl e col ${ }^{(15)}$ demonstraram que a cirurgia inicialmente realizada é o principal fator de prognóstico para os LMS superficiais. Em seu estudo, nenhum dos 14 pacientes tratados com excisão com margens amplas desenvolveu recidiva local ou metástase, enquanto $75 \%$ dos que foram tratados com excisão simples ou enucleação apresentaram recidivas.

A sobrevida em cinco anos varia de $20-74 \%{ }^{(16)}$ e em 10 anos pode atingir $50 \%^{(17,18)}$.

Concluímos então que a excisão com margens amplas é um tratamento adequado para os leiomiosarcomas superficiais. $O$ tratamento radioterápico e quimioterápico está reservado para tumores localmente avançados, persistência ou recidiva. 
ABSTRACT: Leiomyosarcomas are malignant tumors that originate of the smooth muscle. When presents in the perineal region are aggressive and the suitable surgical treatment is not well defined. The authors report the case of a young patient, feminine sex, that presents a perineal nodulation for eight months. At the physical examination was noticed bulging in left perineal region, where a fibroelastic mass of approximately $10 \mathrm{~cm}$ of diameter was palpable. Magnetic nuclear resonance showed voluminous left-sided solid formation of regular contours in perineal region, without signals of perilesional infiltration. The chosed treatment was the excision with ample margins. The patient is currently in ambulatorial accompaniment, without signals of local recurrence.

Key words: Leiomyosarcoma, Rectal Tumors, Perineo, Surgery, Radiotherapy.

\section{REFERÊNCIAS}

1. Grove A, Backman Nohr S. Superficial perineal leiomyosarcoma in na adolescente female and a rewiew of the literature including vulvar leiomyosarcomas. APMIS 1992; 100: p1081-1088.

2. Rosenberg S A, Suit H D, Baker L H Sarcomas of soft tissues. In DeVita V T, Hellman S, Rosenberg S A, editors. Cancer: Principles and practice of oncology. Philadelphia: Lippincott; 1985. p. 1243-1291.

3. Rice J P, MacGillivray D C, Sharpe R W, Weiser E B, Ghosh B C. Perineal Leiomyosarcoma. Gynecol Oncol 1990; 37: p. 132-137.

4. Kessler K J, Kerlakian G M, Welling R E. Perineal and perirectal sarcomas. Dis Colon Rectum 1996; 39(4): p. 468-472.

5. Ramos J R, Pinho M, Ramos R P, Magalhães K M C, Baptista A S. Leiomiossarcoma do reto. Rev Bras Coloproct 1987; 7(3): p. 107-109.

6. Myhre-Jensen O, Hogh J, Ostgaard S E, Nordentoft A M, Sneppen O. Histopathological granding of soft tissue tumours. Prognostic significance in a prospective study of 278 consecutive cases. J Phatol 1991; 163: p. 19-24.

7. Haque S, Dean P J. Stromal neoplasms of the rectum and anal canal. Hum Pathol 1992; 23: p. 762-767.

8. Fields J P, Helwig E B. Leiomyosarcoma of the skin and subcutaneous tissue. Cancer 1981; 47: p. 156-169.

9. Khalifa A A, Bong W L, Rao V K, Williams M J. Leiomyosarcoma of the rectum. Report of a case and rewiew of the literature. Dis Colon Rectum 1986; 29: p. 427-432.

10. Kinsella T J, Sindelar W F, Lack E, Glatstein E, Rosenberg S A. Preliminary results of a randomized study of adjuvant radiation therapy in resectable adult retroperitoneal soft tissue sarcomas. J Clin Oncol 1988; 6: p. 18-25.
11. Glenn J, Sindelar W F, Kinsella T, Glatstein E, Tepper J, Costa J, et al. Results of multimodality therapy of resectable soft-tissue sarcomas of the retroperitoneum. Surgery 1985; 97: p.316-325.

12. Karakousis C P, Velez A F, Emrich L J. Management of retroperitoneal sarcomas and patient survival. Amer J Surg 1985; 150: p. 376-380.

13. Stornes I, Mejlholm I, Jakobsen A. Chemotherapy in perineal leiomyosarcoma. Gynecol Oncol 1992; 44: p.110-112.

14. Choi T K, Ng A, Wong J. Doxorubicin, dacarbazine, vincristine, and cyclophosphamide in the treatment of advanced gastrointestinal leiomyosarcoma. Cancer Treat 1985; 69: p. 443-444.

15. Dahl I, Angervall L. Cutaneous and subcutaneous leiomyosarcoma. A Clinicophathologic study of 47 patients. Path Eur 1974; 9: p. 307-315.

16. Vandoni R E, Givel J C, Essinger A R. Rectal leiomyosarcoma: acute presentation after local injury. Eur J Surg 1992; 158: p. 383-386.

17. Randleman Jr C D, Wolff B G, Dozois R R, Spencer R J, Wieland L H, Ilstrup D M. Leiomyosarcoma of the rectum and anus. A series of 22 cases. Int J Colorect Dis 1989; 4: p. 91-96.

18. Nunes B L B B P, Nossa F L C, Albino P A, Rossoni M D, Formiga G J S, Silva J H. Sarcoma anorretal - Relato de Caso e revisão da literatura. Rev Bras Coloproct 1998;18(3): p.186189.

Endereço para Correspondência:

GALDINO JOSÉ SITONIO FORMIGA

Rua Cônego Xavier, 276

Vila Heliópolis

04231-030 São Paulo/SP

E-mail: gformiga@ig.com.br 